\title{
Numerical modeling of the impact of regenerator housing on the determination of Nusselt numbers
}

Nielsen, Kaspar Kirstein; Nellis, G.F.; Klein, S.A.

Published in:

International Journal of Heat and Mass Transfer

Link to article, DOI:

10.1016/j.jheatmasstransfer.2013.06.032

Publication date:

2013

Document Version

Early version, also known as pre-print

Link back to DTU Orbit

Citation (APA):

Nielsen, K. K., Nellis, G. F., \& Klein, S. A. (2013). Numerical modeling of the impact of regenerator housing on the determination of Nusselt numbers. International Journal of Heat and Mass Transfer, 65, 552-560.

https://doi.org/10.1016/j.ijheatmasstransfer.2013.06.032

\section{General rights}

Copyright and moral rights for the publications made accessible in the public portal are retained by the authors and/or other copyright owners and it is a condition of accessing publications that users recognise and abide by the legal requirements associated with these rights.

- Users may download and print one copy of any publication from the public portal for the purpose of private study or research.

- You may not further distribute the material or use it for any profit-making activity or commercial gain

- You may freely distribute the URL identifying the publication in the public portal 


\title{
Numerical modeling of the impact of regenerator housing on the determination of Nusselt numbers
}

\author{
K.K. Nielsen ${ }^{\mathrm{a}}$, G.F. Nellis ${ }^{\mathrm{b}}$, S.A. Klein ${ }^{\mathrm{b}}$ \\ ${ }^{a}$ Department of Energy Conversion and Storage, Technical University of Denmark, \\ Frederiksborgvej 399, 4000 Roskilde, Denmark \\ kaki@dtu.dk \\ ${ }^{b}$ Department of Mechanical Engineering, University of Wisconsin
}

Engineering Research Building, 1500 Engineering Drive, Madison, WI 53706-1687, USA

\begin{abstract}
It is suggested that the housing of regenerators may have a significant impact when experimentally determining Nusselt numbers at low Reynolds and large Prandtl numbers. In this paper, a numerical model that takes the regenerator housing into account as a domain that is thermally coupled to the regenerator fluid is developed. The model is applied to a range of cases and it is shown that at low Reynolds numbers (well below 100) and at Prandtl numbers appropriate to liquids ( 7 for water) the regenerator housing may influence the experimental determination of Nusselt numbers significantly.

The impact of the housing on the performance during cyclic steady-state regenerator operation is quantified by comparing the regenerator effectiveness for cases where the wall is ignored and with cases where it is included. It is shown that the effectiveness may be decreased by as much as $18 \%$ for the cases considered here. A reduced number of transfer units $\left(\mathrm{NTU}_{\text {eff }}\right)$ is proposed based on the calculated regenerator effectiveness that accounts for the effect of the housing heat capacity.
\end{abstract}

Keywords: Wall effects, numerical modeling, Nusselt number, low Reynolds number, aqueous heat transfer fluid, regenerator

\section{Greek letters}

$\alpha_{\mathrm{w}}$ Thermal diffusivity of the wall $\left[\mathrm{m}^{2} / \mathrm{s}\right]$

$\epsilon$ Regenerator effectiveness 
$\mu_{\mathrm{f}}$ Fluid dynamic viscosity $[\mathrm{Pa} \cdot \mathrm{s}]$

$\psi$ Thermal mass ratio of the wall and the regenerator solid

$\rho$ Mass density $\left[\mathrm{kg} / \mathrm{m}^{3}\right]$

$\tau$ Total cycle time $[\mathrm{s}]$

$\varepsilon$ Bed porosity

$\varphi$ Thermal utilization

\section{Subscripts}

f Fluid index

s Solid index

$\mathrm{w}$ wall index

\section{Variables}

$\Delta p$ Pressure drop $[\mathrm{Pa}]$

$\Delta r_{j}$ Radial extent of the $j$ th cell $[\mathrm{m}]$

$\Delta V_{i, j}$ Volume of the grid cell with indices $i, j\left[\mathrm{~m}^{3}\right]$

$\Delta x$ Axial extent of the cells $[\mathrm{m}]$

$\dot{m}$ Mass flow rate $[\mathrm{kg} / \mathrm{s}]$

$\dot{q}_{\mathrm{fw}, \mathrm{i}}$ The heat flux across the boundary between the fluid and the wall domains at node $\mathrm{i}[\mathrm{W}]$

$\operatorname{Re}_{\mathrm{f}}$ Reynolds number based on the hydraulic diameter

$\operatorname{Re}_{\mathrm{p}}$ Reynolds number based on the sphere diameter

$n_{r, \mathrm{sf}}$ Number of grid points in the $r$-direction in the solid and fluid domains

$T_{\text {hot }}$ Fluid inlet temperature at the hot end $[\mathrm{K}]$

$A=180$, constant in the Ergun equation 
$A_{\mathrm{c}}$ Bed cross sectional area $\left[\mathrm{m}^{2}\right]$

$A_{\mathrm{HT}}$ Total heat transfer surface area of the bed $\left[\mathrm{m}^{2}\right]$

$a_{s}$ Specific surface area $\left[\mathrm{m}^{-1}\right]$

$B=1.8$, constant in the Ergun equation

c Specific heat $[\mathrm{J} / \mathrm{kgK}]$

$d_{h}$ Hydraulic diameter $[\mathrm{m}]$

$d_{p}$ Sphere diameter $[\mathrm{m}]$

$f$ Operating frequency $[\mathrm{Hz}]$

$h$ Convective heat transfer coefficient $[\mathrm{W} /(\mathrm{m} \cdot \mathrm{K})]$

$i, j$ Axial and radial indices, respectively

$k$ Thermal conductivity $[\mathrm{W} /(\mathrm{m} \cdot \mathrm{K})]$

$k_{\text {disp }}$ Thermal dispersion $[\mathrm{W} /(\mathrm{m} \cdot \mathrm{K})]$

$k_{\text {stat }}$ Static thermal conductivity of the bed $[\mathrm{W} /(\mathrm{m} \cdot \mathrm{K})]$

$L$ Length of the regenerator bed $[\mathrm{m}]$

$n$ Index for the timestep

$n_{x}$ Number of grid points in the $x$-direction

$n_{r, \mathrm{w}}$ Number of grid points in the $r$-direction in the wall

$R$ Radius of the regenerator bed $[\mathrm{m}]$

$r$ Radial direction

$r_{j}$ Radial center coordinate of the $j$ th cell $[\mathrm{m}]$

$S_{\mathrm{HT}}$ Total heat transfer surface area of a grid cell $\left[\mathrm{m}^{2}\right]$

$T$ Temperature $[\mathrm{K}]$

$t$ Time $[\mathrm{s}]$ 
$T_{\text {f,cold out }}$ Fluid outlet temperature at the cold end $[\mathrm{K}]$

$u$ Pore fluid velocity $[\mathrm{m} / \mathrm{s}]$

$W$ Width of the wall bed $[\mathrm{m}]$

$x$ Axial direction

CFL Criterium for the timestep

NTU Number of transfer units

$\mathrm{Nu}$ Nusselt number

$h_{\mathrm{w}}$ Inverse thermal resistance between wall and fluid $\left[\mathrm{W} / \mathrm{m}^{2} \mathrm{~K}\right]$

\section{Introduction}

The heat transfer characteristics of packed beds operating at low Reynolds numbers (below 100) and using aqueous heat transfer fluids (with high Prandtl number) are not abundant in literature. Generally, the behavior of regenerators using gases and high Reynolds numbers are the focus of research due to their applications in regenerative cryogenic refrigeration cycles and energy storage. However, certain research areas including near room temperature magnetic refrigeration rely on highly efficient regenerators operating at relatively low Reynolds numbers (ranging between 1 and 100, approximately) using high Prandtl number heat transfer fluids.

It is well known that the heat transfer coefficient, $h$, is a function of the Reynolds number and Prandtl number. It is also apparent that for regenerator geometries based on packed particles (and other similar geometries) the heat transfer coefficient, or Nusselt number, increases as a function of Reynolds number following some power law. However, the experimental determination of the Nusselt number at low Reynolds numbers and using high Prandtl number fluids is experimentally difficult as shown below and not available in detail in the literature.

It is non-trivial to derive accurate heat transfer coefficients from experiments at low Reynolds numbers [1]. Thermal interaction with the ambient (i.e., parasitic losses), axial conduction and the housing of the heat exchanger 
are all issues that can significantly affect the measurements under these conditions. In Ref. [2] a factor as a function of the non-dimensional wall thickness is suggested as a correction for the thermal lag caused by the thermal interaction between the heat exchanger and the surrounding housing / wall.

It is common to apply a numerical model where the heat transfer coefficient may be adjusted in order to match predicted behavior with the experimental data (typically in the form of fluid outlet temperature as a function of time). Techniques for doing this have been applied for decades (see, e.g., Ref. [3]). If the applied numerical model is not sufficiently accurate or if it ignores important physical effects then the resulting $\mathrm{Nu}$-Re correlation may become inaccurate and unphysical; this would be the case if the Nusselt number goes to zero or even becomes negative in the limit when that the Reynolds number approaches zero.

In this paper we propose that the regenerator wall / housing may have an influence on the experimental determination of the heat transfer properties at low Reynolds numbers. That is, the apparent (or measured) heat transfer coefficient may be substantially different from the actual heat transfer coefficient. We also propose that the regenerator wall / housing may have a significant influence on the performance of a regenerator at low Reynolds number. That is, the effectiveness of the regenerator under periodic steadystate operating conditions may be substantially reduced. The wall may act as a passive regenerator surrounding the actual regenerator matrix since heat must be transferred to and from the wall from the regenerator solid and the heat transfer fluid. For housing materials with sufficient thermal diffusivity, axial conduction in the wall may also have an impact on the regenerator performance / apparent heat transfer coefficient.

In order to investigate the effect of the regenerator housing, a detailed numerical model is derived, described, validated and applied to a range of relevant cases. The model is two-dimensional resolving the flow direction (denoted $x$ ) and the transverse direction (denoted $r$ ) while assuming azimuthal symmetry. Three domains are included in the model: the regenerator solid, the heat transfer fluid and the regenerator housing / wall. The appropriate heat transfer equations are solved in all three domains and evolved forward in time. The model is designed so that it may be applied in a single-blow mode, which is relevant if it is used to understand the impact of the housing on the derivation of accurate Nusselt numbers from experimental data of this type. The model can also be used for periodic steady-state operation, i.e. having a periodic (balanced and symmetric) fluid flow. The latter mode is 


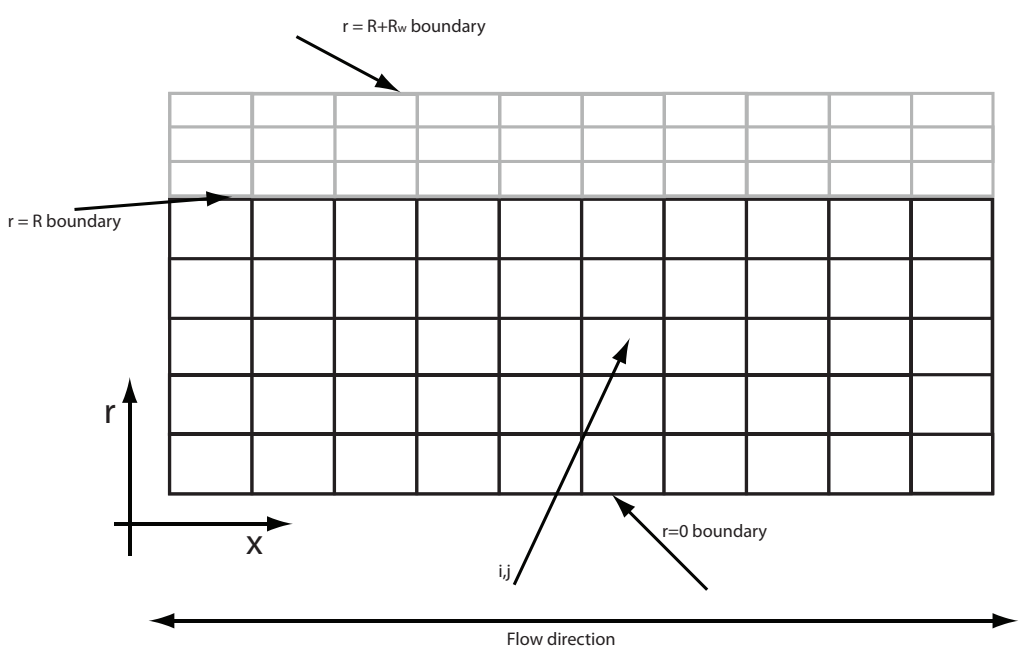

Figure 1: The modeled geometry. The bold part of the model grid indicates the fluid and solid domains. The grey part of the grid indicates the surrounding wall / housing domain. Since the model is radial (with azimuthal symmetry) the volume of the control volume with indices $i, j$ is $\Delta V_{i, j}=2 \pi r_{j} \Delta r_{j} \Delta x$, where the center of the cell in the radial direction is denoted $r_{j}$.

relevant when probing the impact of the housing on regenerator performance, or effectiveness, as a function of operating conditions and wall properties.

The remainder of this paper is outlined as follows. In Sec. 2 the numerical model is derived and presented. In Sec. 3 the results are presented. Finally, in Sec. 4 the results are discussed and the paper is concluded.

\section{Numerical model}

The modeled geometry is cylindrical and assumes symmetry around the center axis. The axial $(x)$ and the radial $(r)$ directions are spatially resolved in all three domains: fluid, solid regenerator matrix and the solid wall, respectively. Figure 1 shows a schematic of the model geometry and defines the coordinate system. In the following section the governing equations for each of the three domains are written out in discretized form using finite differences. 


\subsubsection{Governing regenerator equations}

The model solves the transient partial differential equations describing heat transfer via conduction and convection in a porous regenerator:

$$
\begin{aligned}
\rho_{\mathrm{f}} c_{\mathrm{f}} \varepsilon\left(\frac{\partial T_{\mathrm{f}}}{\partial t}+u \frac{\partial T_{\mathrm{f}}}{\partial x}\right)= & \frac{1}{r} \frac{\partial}{\partial r}\left(k_{\mathrm{disp}, \mathrm{r}} r \frac{\partial T_{\mathrm{f}}}{\partial r}\right)+\frac{\partial}{\partial x}\left(k_{\mathrm{disp}, \mathrm{x}} \frac{\partial T_{\mathrm{f}}}{\partial x}\right)- \\
& h a_{s}\left(T_{\mathrm{f}}-T_{\mathrm{s}}\right)+\left|\frac{\Delta p \dot{m}}{\rho_{\mathrm{f}} L A_{\mathrm{c}}}\right| \\
\rho_{\mathrm{s}} c_{\mathrm{s}}(1-\varepsilon) \frac{\partial T_{\mathrm{s}}}{\partial t}= & \frac{1}{r} \frac{\partial}{\partial r}\left(k_{\mathrm{stat}} r \frac{\partial T_{\mathrm{s}}}{\partial r}\right)+\frac{\partial}{\partial x}\left(k_{\mathrm{stat}} \frac{\partial T_{\mathrm{s}}}{\partial x}\right)+ \\
\rho_{\mathrm{w}} c_{\mathrm{w}} \frac{\partial T_{\mathrm{w}}}{\partial t}= & \frac{1}{r} \frac{\partial}{\partial r}\left(k_{\mathrm{w}} r \frac{\partial T_{\mathrm{w}}}{\partial r}\right)+\frac{\partial}{\partial x}\left(k_{\mathrm{w}} \frac{\partial T_{\mathrm{w}}}{\partial x}\right)
\end{aligned}
$$

The temperature fields $(T)$ are solved for on the three domains (fluid, regenerator solid and wall, respectively denoted by subscripts $\mathrm{f}, \mathrm{s}$ and $\mathrm{w}$ ). The fluid and solid domains are coupled through the convective heat transfer coefficient, $h$ and the specific heat transfer surface area, $a_{s}$, of the solid regenerator material.

The above given equations for the fluid and the solid (1-2) are volume averaged since the actual porous medium is not resolved. For a given control volume a fraction is fluid and the rest of the volume is solid. This is modeled through the porosity, $\varepsilon$. The volume average approach also introduces dispersion coefficients $\left(k_{\text {disp }}\right)$. These and the remaining terms in Eqs. 1-3 are explained in detail in the following.

\subsection{Governing discretized equations}

Letting the index $n$ denote the timestep and $i$ and $j$ the spatial grid indices in the $x$ - and $r$-directions, respectively, the energy balance for the discretized fluid domain may be written in the following way:

$$
\begin{array}{r}
\underbrace{\rho_{\mathrm{f}, i, j} c_{\mathrm{f}, i, j} \varepsilon 2 \pi r_{j} \Delta r_{j} \Delta x}_{\text {Thermal mass of node } i, j}(\underbrace{\frac{T_{\mathrm{f}, i, j}^{n+1}-T_{\mathrm{f}, i, j}^{n}}{\Delta t}}_{\text {Energy storage }}+\underbrace{u_{i, j} \frac{T_{\mathrm{f}, i+1, j}^{n+1}-T_{\mathrm{f}, i-1, j}^{n+1}}{2 \Delta x}}_{\text {Convection }}) \\
\underbrace{\frac{T_{\mathrm{f}, i, j+1}^{n+1}-T_{\mathrm{f}, i, j}^{n+1}}{2 \pi\left(r_{j}+\Delta r_{j} / 2\right) \Delta x}\left(\frac{\Delta r_{j+1} / 2}{k_{\mathrm{disp}, \mathrm{r}, i, j+1}}+\frac{\Delta r_{j} / 2}{k_{\mathrm{disp}, \mathrm{r}, i, j}}\right)}_{\text {Conduction between nodes } i, j \text { and } i, j+1}-\underbrace{\frac{T_{\mathrm{f}, i, j}^{n+1}-T_{\mathrm{f}, i, j-1}^{n+1}}{\frac{1}{2 \pi\left(r_{j}-\Delta r_{j} / 2\right) \Delta x}\left(\frac{\Delta r_{j} / 2}{k_{\mathrm{disp}, \mathrm{r}, i, j}}+\frac{\Delta r_{j-1} / 2}{k_{\mathrm{dis}, \mathrm{r}, i, j-1}}\right)}}_{\text {Conduction between nodes } i, j \text { and } i, j-1}+
\end{array}
$$




$$
\begin{gathered}
\frac{T_{\mathrm{f}, i+1, j}^{n+1}-T_{\mathrm{f}, i, j}^{n+1}}{\underbrace{\frac{\Delta x / 2}{2 \pi r_{j} \Delta r_{j}}\left(\frac{1}{k_{\mathrm{disp}, \mathrm{x}, i+1, j}}+\frac{1}{k_{\mathrm{disp}, \mathrm{x}, i, j}}\right)}_{\text {Conduction between nodes } i+1, j \text { and } i, j}-\underbrace{\frac{\Delta x / 2}{2 \pi r_{j} \Delta r_{j}}\left(\frac{1}{k_{\mathrm{disp}, \mathrm{x}, i, j}}+\frac{1}{k_{\mathrm{disp}, \mathrm{x}, i-1, j}}\right)}_{\text {Conduction between nodes } i, j \text { and } i-1, j}}- \\
\underbrace{h S_{\mathrm{HT}}\left(T_{\mathrm{f}, i, j}^{n+1}-T_{\mathrm{s}, i, j}^{n+1}\right)}_{\text {Heat transfer between fluid and solid }}+\underbrace{\left|\frac{\Delta p m}{\rho_{\mathrm{f}}} \frac{2 \pi r_{j} \Delta r_{j} \Delta x}{L A_{\mathrm{c}}}\right|}_{\text {Viscous dissipation }} \mid
\end{gathered}
$$

Here $\rho$ and $c$ denote the density and specific of the solid material and fluid, and the subscripts $\mathrm{f}$ and s denote the fluid and solid, respectively. The conductivity, $k_{\text {disp }}$, of the fluid includes dispersion, which may differ depending on whether the radial or the axial direction is considered (see Sec. 2.5). The porosity of the bed is $\varepsilon$ and the heat transfer coefficient describing the fluidsolid thermal interaction is denoted $h$. The heat transfer surface area of the solid within the cell is $S_{\mathrm{HT}}$. The total pressure drop across the bed is $\Delta p$ and the mass flow rate is $\dot{m}$. $T^{n}$ is the temperature at time $t_{n}$ and $T^{n+1}$ is the temperature at time $t_{n}+\Delta t$. Finally, the bed cross sectional area is $A_{\mathrm{c}}$

The equation for the regenerator solid may be formulated as

$$
\begin{aligned}
& \underbrace{\rho_{\mathrm{s}, i, j} c_{\mathrm{s}, i, j}(1-\varepsilon) 2 \pi r_{j} \Delta r_{j} \Delta x}_{\text {Thermal mass of node } i, j} \underbrace{\frac{T_{\mathrm{s}, i, j}^{n+1}-T_{\mathrm{s}, i, j}^{n}}{\Delta t}}_{\text {Energy storage }}= \\
& \underbrace{\frac{T_{\mathrm{s}, i, j+1}^{n+1}-T_{\mathrm{s}, i, j}^{n+1}}{2 \pi\left(r_{j}+\Delta r_{j} / 2\right) \Delta x}\left(\frac{\Delta r_{j+1} / 2}{k_{\mathrm{stat}, i, j+1}}+\frac{\Delta r_{j} / 2}{k_{\mathrm{stat}, i, j}}\right)}_{\text {Conduction between nodes } i, j \text { and } i, j+1}-\underbrace{\frac{1}{2 \pi\left(r_{j}-\Delta r_{j} / 2\right) \Delta x}\left(\frac{\Delta r_{j} / 2}{k_{\mathrm{stat}, i, j}}+\frac{\Delta r_{j-1} / 2}{k_{\mathrm{stat}, i, j-1}}\right)}_{\text {Conduction between nodes } i, j \text { and } i, j-1}+
\end{aligned}
$$

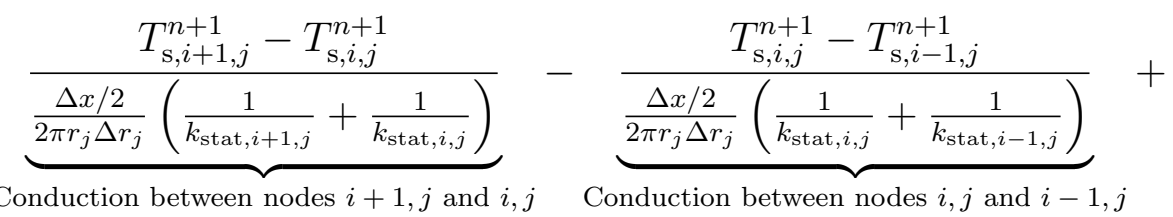

$$
\begin{aligned}
& \underbrace{h S_{\mathrm{HT}}\left(T_{\mathrm{f}, i, j}^{n+1}-T_{\mathrm{s}, i, j}^{n+1}\right)}
\end{aligned}
$$

The conductivity of the solid is denoted $k_{\text {stat }}$, which is the effective conductivity of the whole bed when there is no fluid movement, as discussed in Sec. 
2.4. Finally the equation for the solid wall is

$$
\begin{aligned}
& \underbrace{\rho_{\mathrm{w}, i, j} c_{\mathrm{w}} 2 \pi r_{j} \Delta r_{j} \Delta x}_{\text {Thermal mass of node } \mathrm{i}, \mathrm{j}} \underbrace{\frac{T_{\mathrm{w}, i, j}^{n+1}-T_{\mathrm{w}, i, j}^{n}}{\Delta t}}_{\text {Energy storage }}= \\
& \underbrace{\frac{T_{\mathrm{w}, i, j+1}^{n+1}-T_{\mathrm{w}, i, j}^{n+1}}{2 \pi\left(r_{j}+\Delta r_{j} / 2\right) \Delta x}\left(\frac{\Delta r_{j+1} / 2}{k_{\mathrm{w}, i, j+1}}+\frac{\Delta r_{j} / 2}{k_{\mathrm{w}, i, j}}\right)}_{\text {Conduction between nodes } \mathrm{i}, \mathrm{j} \text { and } \mathrm{i}, \mathrm{j}+1}-\underbrace{\frac{1}{2 \pi\left(r_{j}-\Delta r_{j} / 2\right) \Delta x}\left(\frac{\Delta r_{j} / 2}{k_{\mathrm{w}, i, j}}+\frac{\Delta r_{j-1} / 2}{k_{\mathrm{w}, i, j-1}}\right)}_{\text {Conduction between nodes } \mathrm{i}, \mathrm{j} \text { and } \mathrm{i}, \mathrm{j}-1}+ \\
& \underbrace{\frac{T_{\mathrm{w}, i+1, j}^{n+1}-T_{\mathrm{w}, i, j}^{n+1}}{\frac{\Delta x / 2}{2 \pi r_{j} \Delta r_{j}}\left(\frac{1}{k_{\mathrm{w}, i+1, j}}+\frac{1}{k_{\mathrm{w}, i, j}}\right)}}_{\text {Conduction between nodes } \mathrm{i}+1, \mathrm{j} \text { and } \mathrm{i}, \mathrm{j}}-\underbrace{\frac{T_{\mathrm{w}, i, j}^{n+1}-T_{\mathrm{w}, i-1, j}^{n+1}}{\frac{\Delta x / 2}{2 \pi r_{j} \Delta r_{j}}\left(\frac{1}{k_{\mathrm{w}, i, j}}+\frac{1}{k_{\mathrm{w}, i-1, j}}\right)}}_{\text {Conduction between nodes } \mathrm{i}, \mathrm{j} \text { and } \mathrm{i}-1, \mathrm{j}}
\end{aligned}
$$

The conductivity of the wall is $k_{\mathrm{w}}$. For all three domains the index $i$ runs from 2 to $n_{x}-1$. In the fluid and solid regenerator domains, the index $j$ runs from 2 to $n_{r, \mathrm{sf}}-1$ whereas in the wall domain it runs from $n_{r, \mathrm{sf}}+2$ to $n_{r, \mathrm{sf}}+n_{r, \mathrm{w}}-1$. At $i=1, n_{x}$ and $j=1, n_{r, \mathrm{sf}}, n_{r, \mathrm{sf}}+1$, respectively, the equations are altered in order to take the appropriate boundary conditions into account (see Sec. 2.2).

The thermal properties (mass density, specific heat and conductivity) are assumed to be functions of the temperature at the beginning of each timestep $n$, i.e. they are explicitly prescribed in each timestep.

The discretized formulation of the regenerator equations given above is implicit. The algorithm for solving the equations is described in Sec. 2.3.

\subsection{Boundary conditions}

The coupling between the fluid/solid and the wall domains is determined assuming that the fluid (at node $j=n_{r, \mathrm{sf}}$ ) is in contact with the wall (at node $j=n_{r, \mathrm{sf}}+1$ ) through the following equation (where $1 \leq i \leq n_{x}$ )

$$
\dot{q}_{\mathrm{fw}, i}=\frac{T_{\mathrm{w}, i, 1+n_{r, \mathrm{sf}}}^{n+1}-T_{\mathrm{f}, i, n_{r, \mathrm{sf}}}^{n+1}}{\frac{1}{2 \pi\left(r_{n_{r, \mathrm{sf}}}+\Delta r_{n_{r, \mathrm{sf}}} / 2\right) \Delta x}\left(\frac{\Delta r_{1+n_{r, \mathrm{sf}}} / 2}{k_{\mathrm{w}, i, 1+n_{r, \mathrm{sf}}}}+\frac{\Delta r_{n_{r, \mathrm{sf}}} / 2}{k_{\mathrm{f}, i, n_{r, \mathrm{sf}}}}+\frac{1}{h_{\mathrm{w}}}\right)}
$$

This term is added to the right hand side of the fluid equation (4) and subtracted from the right hand side of the wall equation (6) at the relevant values of $j$. The third term in the denominator of Eq. 7 represents a thermal 
resistance (per surface area) between the two domains. If $h_{\mathrm{w}} \rightarrow 0$ then the two domains will be decoupled and in the limit when $h_{\mathrm{w}} \rightarrow \infty$ there is no thermal resistance between the two domains other than what may be deemed the intrinsic physical thermal resistance (the two first terms in the denominator of Eq. 7). The result presented in the following are all computed assuming that $h_{\mathrm{w}}=\infty$. The fluid thermal conductivity in Eq. 7 is assumed to be the intrinsic fluid conductivity (i.e. $k_{\mathrm{f}}$ and $n o t k_{\mathrm{disp}, \mathrm{r}}$ ). This term (Eq. 7) thus represents the boundary condition for $T_{\mathrm{f}}$ and $T_{\mathrm{w}}$ at $r=R$. The boundary condition for $T_{\mathrm{s}}$ at $r=R$ is assumed adiabatic and is thus:

$$
\left.\frac{\partial T_{\mathrm{s}}}{\partial r}\right|_{r=R}=0
$$

The boundaries at $r=0$ and $r=R+W$ are assumed adiabatic ( $W$ denotes the thickness of the wall), i.e.:

$$
\begin{aligned}
\left.\frac{\partial T_{\mathrm{f}}}{\partial r}\right|_{r=0} & =0 \\
\left.\frac{\partial T_{\mathrm{s}}}{\partial r}\right|_{r=0} & =0 \\
\left.\frac{\partial T_{\mathrm{w}}}{\partial r}\right|_{r=R+W} & =0 .
\end{aligned}
$$

At the two ends of the regenerator $(x=0$ and $x=L$, respectively) the boundary conditions for the fluid depend on the direction of the flow:

$$
\begin{aligned}
\left.\frac{\partial T_{\mathrm{f}}}{\partial x}\right|_{x=0} & =0 \text { if } \dot{m} \leq 0 \\
\left.T_{\mathrm{f}}\right|_{x=0} & =T_{0} \text { if } \dot{m}>0 \\
\left.\frac{\partial T_{\mathrm{f}}}{\partial x}\right|_{x=L} & =0 \text { if } \dot{m} \geq 0 \\
\left.T_{\mathrm{f}}\right|_{x=L} & =T_{1} \text { if } \dot{m}<0
\end{aligned}
$$

Since the Péclet number is always significantly greater than one ( $\mathrm{Pe}=$ $\left.\mathrm{Re}_{\mathrm{f}} \mathrm{Pr}\right)$, where $\mathrm{Re}_{\mathrm{f}}$ and $\mathrm{Pr}$ are the Reynolds and Prandtl numbers, respectively, the above given inlet boundary conditions (Eqs. 13 and 15) are assumed valid and diffusion at the inlets is ignored (see Refs. [2, 4] for details about diffusion at inlet boundaries). 
Finally, the boundary conditions for the regenerator solid and the wall at $x=0$ and $x=L$ are

$$
\begin{aligned}
& \left.\frac{\partial T_{\mathrm{s}}}{\partial x}\right|_{x=0, x=L}=0 \\
& \left.\frac{\partial T_{\mathrm{w}}}{\partial x}\right|_{x=0, x=L}=0
\end{aligned}
$$

When simulating a single blow the direction of the flow is constant whereas when simulating periodic steady-state operating the flow direction is alternating periodically thus making the boundary conditions in Eqs. 13-15 vary in time.

\subsection{Numerical solution of the discretized equations}

The discretized equations, 4-6, are formulated and solved using the fully implicit method. Great care should thus be taken when choosing the timestep and grid size in order to achieve an accurate solution. Given a specified grid size, the following criterion defines the timestep size:

$$
\mathrm{CFL}=\frac{\dot{m} \Delta t}{\rho_{\mathrm{f}} \pi R^{2} \varepsilon \Delta x}
$$

where the radius of the bed is $R$. The CFL (Courant-Friedrichs-Lewy) number may be set to any value due to the implicit solver. It was, however, found through thorough testing that a number of spatial nodes of 150, corresponding to a grid resolution of less than $1 \mathrm{~mm}$ in the $\mathrm{x}$-direction for the cases studied here, and CFL $=0.1$ gives consistent and precise results.

The model was compared to the classical Schumann solution [5] in singleblow mode and found to agree (when neglecting axial conduction, viscous dissipation and internal gradients in the solid) to within $0.3 \%$ when applying the above mentioned spatial and temporal resolution.

The model was also tested against the published regenerator effectiveness solution given by Dragutinovic and Baclic [6] under the assumptions that there is zero entrained fluid heat capacity, no axial conduction, no dispersion, no viscous dissipation and all the thermal properties are constant. In this mode the model is run with periodic boundary conditions.

It was found that at values of the NTU (defined below) from 0 to about 1000 the model agrees to within $0.3 \%$ with the published solution at relevant 
values of the thermal utilization (ranging from 0.2 to 1.5 and further discussed in Sec. 3) in the worst case.

The solution is implemented in Fortran and the source code is freely available online [7].

\subsection{Correlations}

The correlations required to run the model are discussed in this section.

The heat transfer coefficient, $h$, is found from the Nusselt number through

$$
\mathrm{Nu}=\frac{h d_{h}}{k_{\mathrm{f}}} .
$$

It is important to note that it is the fluid thermal conductivity, $k_{\mathrm{f}}$, that enters Eq. 19 and not, e.g., the dispersion corrected conductivity $\left(k_{\text {disp }}\right)$. The hydraulic diameter, $d_{h}$, for packed spheres is given by

$$
d_{h}=\frac{2}{3} \frac{\varepsilon}{1-\varepsilon} d_{p}
$$

where the sphere diameter is $d_{p}$. The specific surface area is

$$
a_{s}=6 \frac{1-\varepsilon}{d_{p}} .
$$

There are numerous correlations for the Nusselt number as a function of the Reynolds and Prandtl numbers. An example of such a correlation was given in Ref. [8]:

$$
\mathrm{Nu}=2+1.1 \operatorname{Re}_{p}^{0.6} \operatorname{Pr}^{1 / 3}
$$

and is referred to in the following as the "Wakao and Kaguei" relation. It is seen that in the limit $\operatorname{Re} \rightarrow 0$ the Nusselt number becomes 2, which is the conduction limit for a single sphere [9]. The correlation given in Eq. 22 is a function of the particle size-based Reynolds number:

$$
\operatorname{Re}_{p}=\frac{\rho_{\mathrm{f}} u \varepsilon d_{p}}{\mu_{\mathrm{f}}}
$$

where the pore velocity is denoted $u$ and the dynamic viscosity of the fluid is $\mu_{\mathrm{f}}$. The Reynolds number based on hydraulic diameter is then

$$
\operatorname{Re}_{\mathrm{f}}=\frac{\rho_{\mathrm{f}} u \varepsilon d_{h}}{\mu_{\mathrm{f}}}=\frac{2}{3} \frac{\varepsilon}{1-\varepsilon} \operatorname{Re}_{p} .
$$


The mass flow rate of the regenerator is

$$
\dot{m}_{\mathrm{f}}=\rho_{\mathrm{f}} u \varepsilon A_{\mathrm{c}} .
$$

Finally, the pressure drop across the bed is calculated using the Ergun relation [10]:

$$
\begin{aligned}
\frac{\mathrm{d} p}{\mathrm{~d} x} & =\frac{A \alpha \mu_{\mathrm{f}} \varepsilon u}{d_{p}^{2}}+\frac{B \beta \rho_{\mathrm{f}}(\varepsilon u)^{2}}{d_{p}} \\
\alpha & =\frac{(1-\varepsilon)^{2}}{\varepsilon^{2}} \\
\beta & =\frac{1-\varepsilon}{\varepsilon^{3}} \\
A=180, \quad B=1.8 . &
\end{aligned}
$$

\subsection{The effective conductivity of the regenerator}

The model presented above is based on volume averages where the control volume includes one or multiple spheres and fluid paths. It is therefore not sufficient to consider the intrinsic thermal conductivity of the regenerator solid and fluid, respectively, when determining the axial and radial conduction in the bed. The phenomena known as dispersion and the effective static conduction of the bed must be taken into account. As shown above in Eqs. 1-2 the static conductivity is used to compute conduction in the regenerator solid energy balance whereas the dispersion-related conductivity is assumed in the fluid equation. Several correlations for these quantities exist in literature (see, e.g., Refs. [11, 12] for detailed discussions). The correlation for the static conductivity applied in this work is taken from Ref. [13]:

$$
\begin{array}{r}
k_{\text {stat }}=k_{\mathrm{f}}\left[\left(1-\alpha_{0}\right) \frac{\varepsilon f_{0}+k_{\mathrm{s}} / k_{\mathrm{f}}\left(1-\varepsilon f_{0}\right)}{1-\varepsilon\left(1-f_{0}\right)+k_{\mathrm{s}} / k_{\mathrm{f}} \varepsilon\left(1-f_{0}\right)}+\right. \\
\left.\alpha_{0} \frac{2\left(k_{\mathrm{s}} / k_{\mathrm{f}}\right)^{2}(1-\varepsilon)+(1+2 \varepsilon) k_{\mathrm{s}} / k_{\mathrm{f}}}{(2+\varepsilon) k_{\mathrm{s}} / k_{\mathrm{f}}+1-\varepsilon}\right] \\
f_{0}=0.8+0.1 \varepsilon \\
\log \alpha_{0}=-4.898 \varepsilon, 0 \leq \varepsilon \leq 0.0827 \\
\log \alpha_{0}=-0.405-3.154(\varepsilon-0.0827), 0.0827 \leq \varepsilon \leq 0.298 \\
\log \alpha_{0}=-1.084-6.778(\varepsilon-0.298), 0.298 \leq \varepsilon \leq 0.580 .
\end{array}
$$


The fluid dispersion in the axial direction (for uniformly packed spheres) is given by [11]:

$$
k_{\text {disp }, \mathrm{x}}=3 / 4 k_{\mathrm{f}} \varepsilon \operatorname{Re}_{\mathrm{f}} \operatorname{Pr} .
$$

The expression in Eq. 35 is only valid when the Reynolds number is greater than 10 [14]. When $\operatorname{Re}_{\mathrm{f}} \leq 1$ the thermal conductivity entering Eq. 1 is simply the fluid intrinsic conductivity. In the range where $1<\operatorname{Re}_{\mathrm{f}}<10$ a linear variation between $k_{\mathrm{f}}$ and the expression in Eq. 35 is assumed.

The dispersion in the radial direction, $k_{\text {disp,r }}$, is assumed to be $1 / 5$ of the dispersion in the axial direction [14] although at the low Reynolds numbers it becomes equal to the fluid intrinsic conductivity, $k_{\mathrm{f}}$. Finally, it is noted that when there is no flow, $R e=0$, the dispersive coefficient in Eq. 35 is zero and thus only static conduction is present in the bed as described in Eq. 30 .

\section{Results}

The results presented in this section are divided in three sets. In Sec. 3.1 the model is used to simulate a single blow experiment where the wall is taken into account. This simulated data set is then compared to model results where the wall is not taken into account but the Nusselt number is varied to match the simulated data. This simulates the process of using single blow experimental data in order to infer a heat transfer coefficient.

In Secs. 3.2-3.3 the regenerator effectiveness computed in the case where the regenerator housing is neglected is compared to cases with different housing materials and geometries. The regenerator effectiveness is computed for the periodic steady-state solution. Finally, in Sec. 3.4 a corrected NTU (denoted $\mathrm{NTU}_{\text {eff }}$ ) that accounts for the effect of the housing heat capacity on the effectiveness is suggested.

The non-dimensional groups that are used for analysis of the results based on the effectiveness under periodic steady state include the thermal utilization $(\varphi)$ and the number of transfer units (NTU):

$$
\begin{aligned}
\varphi & =\frac{\dot{m} c_{\mathrm{f}}}{2 f m_{\mathrm{s}} c_{\mathrm{s}}} \\
\mathrm{NTU} & =\frac{h A_{\mathrm{HT}}}{\dot{m} c_{\mathrm{f}}} .
\end{aligned}
$$

The operating frequency of the regenerator is $f=1 / \tau$ where $\tau$ is the total cycle time and $A_{\mathrm{HT}}$ denotes the total heat transfer surface area of the 
Table 1: The applied thermal properties of the three domains resolved in the model (regenerator solid, heat transfer fluid and regenerator wall, respectively). The properties reflect gadolinium $(\mathrm{Gd})$, water and various materials for the wall.

\begin{tabular}{c|cccc}
\hline Property & $k[\mathrm{~W} /(\mathrm{m} \cdot \mathrm{K})]$ & $\rho\left[\mathrm{kg} / \mathrm{m}^{3}\right]$ & $c[\mathrm{~J} /(\mathrm{kg} \cdot \mathrm{K})]$ & $\mu_{\mathrm{f}}[\mathrm{Pa} \cdot \mathrm{s}]$ \\
\hline Solid & 10.5 & 7900 & 300 & - \\
\hline Fluid & 0.6 & 1000 & 4200 & 0.001 \\
\hline Wall & $0.25,1,5,10,15,20,100,240$ & 1000 & 1500 & - \\
\hline
\end{tabular}

regenerator solid. The utilization describes the ratio between the thermal mass of fluid moved through the regenerator to the total thermal mass of the regenerator solid. The NTU describes the ratio of the amount of heat transferred between the solid and the fluid to the thermal mass of the fluid moved.

The ratio between thermal mass of the wall and the thermal mass of the regenerator solid is another dimensionless number used in the analysis:

$$
\psi=\frac{m_{\mathrm{w}} c_{\mathrm{w}}}{m_{\mathrm{s}} c_{\mathrm{s}}} .
$$

The regenerator effectiveness is defined as the ratio of the amount of heat that is actually transferred during a blow process between the solid and the fluid to the maximum possible heat transfer:

$$
\epsilon=\frac{\int_{0}^{\tau / 2}\left(T_{\text {hot }}-T_{\text {f,cold out }}\right) \mathrm{d} t}{\left(T_{\text {hot }}-T_{\text {cold }}\right) \tau / 2} .
$$

Constant thermal properties are assumed throughout all simulations. Hot fluid enters at one end (at temperature $T_{\text {hot }}$ ) and exits at the other (cold) end at temperature $T_{\mathrm{f}, \text { cold out }}$.

The properties of the three domains are provided in Table 1. The regenerator solid resembles gadolinium (which is a commonly applied material in active magnetic regenerators) and the heat transfer fluid resembles water. The wall conductivity spans several orders of magnitude in order to evaluate the impact of this parameter on performance. Considering Eq. 3 it is clear that the central parameters are the product of the mass density and the specific heat $(\rho c)$ and the thermal conductivity. These have been chosen so they resemble materials such as plastic nylon, stainless steel and aluminum. The geometric parameters applied in the model are provided in Table 2 . It is noted that the parameter $h_{\mathrm{w}}$ is set to infinity, i.e. the coupling between the 
Table 2: The geometric and operating properties applied in the model.

\begin{tabular}{c|cc}
\hline Property & Value & Description \\
\hline$L$ & $0.1 \mathrm{~m}$ & Regenerator length \\
\hline$R$ & $8.3 \mathrm{~mm}$ & Regenerator radius \\
\hline$W$ & $1-3 \mathrm{~mm}$ & Wall thickness \\
\hline$d_{p}$ & $0.5 \mathrm{~mm}$ & Sphere diameter \\
\hline$\varepsilon$ & 0.36 & Porosity assumed in the regenerator (closely packed spheres) \\
\hline$n_{x}$ & 150 & Resolution in the axial direction \\
\hline$n_{r, \mathrm{sf}}$ & 10 & Radial resolution in the regenerator \\
\hline$n_{r, \mathrm{w}}$ & 10 & Radial resolution in the wall \\
\hline$T_{\text {init }}$ & $290 \mathrm{~K}$ & Initial temperature of all domains \\
\hline$T_{\text {inlet }}$ & $300 \mathrm{~K}$ & Cold side temperature (periodic steady-state simulations) \\
\hline$T_{\text {cold }}$ & $290 \mathrm{~K}$ & Hot side temperature (periodic steady-state simulations) \\
\hline$T_{\text {hot }}$ & $300 \mathrm{~K}$ & Range of operating frequencies at a utilization of 0.5 \\
\hline$R_{\mathrm{f}}$ & $0.86-86.8$ & Range of fluid-based Reynolds numbers applied in the simulations \\
\hline$f(\varphi=0.5)$ & $0.128-12.8$ & Range of operating frequencies at a utilization of 1.5 \\
\hline$f(\varphi=1.5)$ & $0.042-4.27$ &
\end{tabular}

fluid and the wall domains is dominated by the intrinsic thermal resistance as defined in Eq. 7.

\subsection{Single blow analysis of wall impact at low Re}

A typical method applied for determining the convective heat transfer coefficient in a single-blow experiment is to compare the outlet temperature curves measured in the experiment with a suitable model. The heat transfer coefficient used by the model is adjusted in order to provide the best agreement. This comparison may be done in several ways (as described in detail by Heggs and Burns [3]). Each of the different methods use some characteristic of the outlet temperature curves for the comparison. This may, for example, be the maximum value of the gradient of the outlet temperature with respect to time, the root-mean-square difference between the curves or the difference between the time that the temperature change reaches $20 \%$ and $80 \%$ (defined relative to the initial and inlet temperatures). The value of the heat transfer coefficient, or the Nusselt number, is varied in the model to provide the best match to the experimental data.

In this work a simulated experimental data set has been produced by simulating a single-blow with the model while the wall is enabled (having 


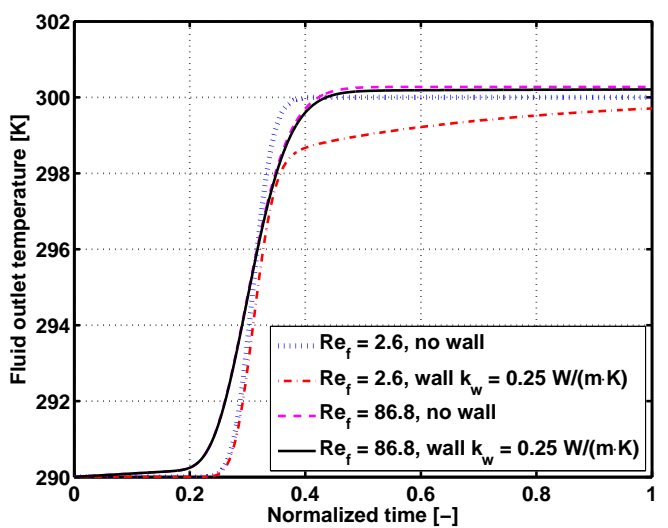

(a)

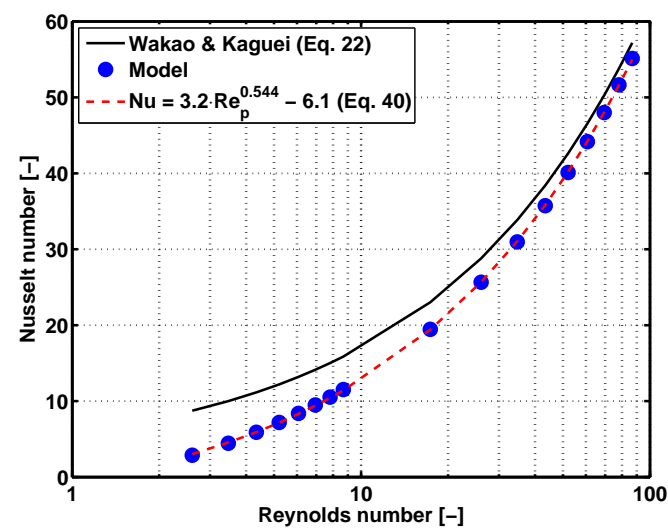

(b)

Figure 2: (Color online) (a) The fluid outlet temperature as a function of normalized time for the case with no wall and that with a wall of low thermal conductivity. $R_{\mathrm{f}}=$ 2.6 and $86.8, T_{\text {init }}=290 \mathrm{~K}, T_{\text {inlet }}=300 \mathrm{~K}$, wall thickness was $1 \mathrm{~mm}$. (b) The Nusselt number as a function of hydraulic diameter based Reynolds number.

thermal conductivity $\left.k_{\mathrm{w}}=0.25 \mathrm{~W} /(\mathrm{m} \cdot \mathrm{K})\right)$. This results in an outlet fluid temperature profile as a function of time denoted $T_{\mathrm{f}, \mathrm{w}, \mathrm{out}}(t)$. The model is then applied with the same geometry and operating conditions but with the wall disabled; the fluid temperature outlet curve is denoted $T_{\mathrm{f}, \mathrm{nw} \text {,out }}(t)$. The Nusselt-number is varied in the model until the two temperature profiles match as closely as possible using some criteria. This process represents the typical technique used to analyze experimental data in a single blow case. Figure 2(a) shows examples of such outlet curves for a low and a high Reynolds number.

The resulting Nusselt number is plotted as a function of Reynolds number in Fig. 2(b). For comparison, the relation applied in the simulated data (where the wall was enabled) is plotted in the same figure and denoted "Wakao \& Kaguei" thus following Eq. 22. The model data that matches the simulated data is fitted to a power law resulting in:

$$
\mathrm{Nu}_{\text {fit }}=3.2 \operatorname{Re}_{\mathrm{p}}^{0.544}-6.1 \text {, }
$$

in which the fitted parameters 3.2, 0.544 and 6.1 have $95 \%$ confidence intervals of $0.1,0.007$ and 0.4 , respectively. Two points are important to stress here. Firstly, as the Reynolds number decreases the difference (both absolute and relative) between the applied relation (Eq. 22) and the fitted data 


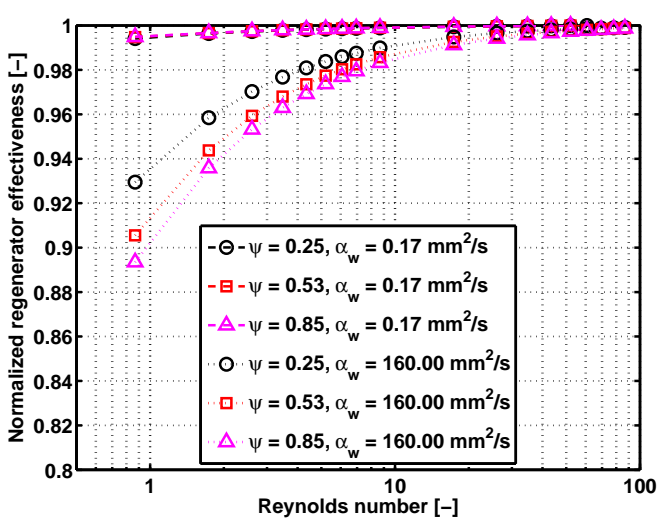

(a) $\varphi=0.5$

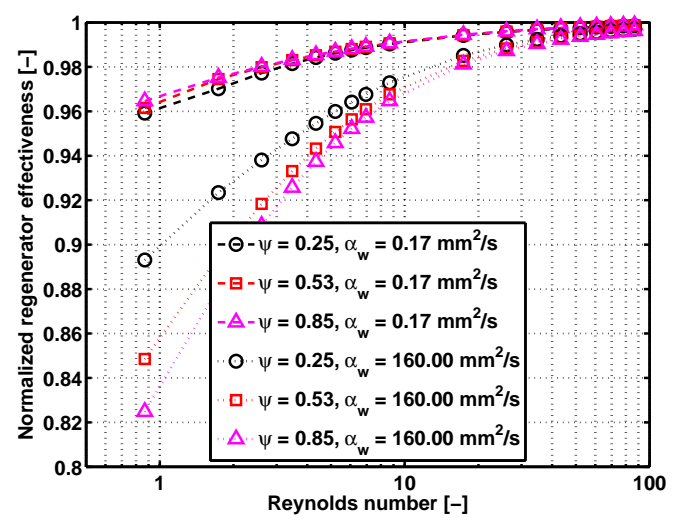

(b) $\varphi=1.5$

Figure 3: (Color online) The regenerator effectiveness (defined in Eq. 39) as a function of the hydraulic diameter based Reynolds number and normalized to the no wall case. The legend refers to cases with different wall thicknesses expressed as a ratio between the thermal mass of the wall and the thermal mass of the solid and different values of the thermal diffusivity of the wall $\left(\alpha_{\mathrm{w}}\right)$.

(Eq. 40) increases. Secondly, in the limit where $R e \rightarrow 0$ the Nusselt number that best matches the data becomes negative, which is unphysical. It should rather approach the conduction limit, i.e. some positive value. This result indicates that the influence of the wall has a significant impact on the determination of heat transfer coefficients at low Reynolds numbers using a single blow experiment.

\subsection{Effectiveness as a function of Reynolds number}

The normalized regenerator effectiveness is defined as the ratio of the effectiveness of the regenerator operating under periodic steady state conditions to the effectiveness of the regenerator neglecting the impact of the wall. The normalized regenerator effectiveness is given as a function of the Reynolds number (based on hydraulic diameter) in Fig. 3. The different graphs represent the variation in $\psi$ and the variation in wall thermal diffusivity $\left(\alpha_{\mathrm{w}}=\frac{k_{\mathrm{w}}}{\rho_{\mathrm{w}} c_{\mathrm{w}}}\right)$ as described in the figure legend.

The trends in the figure are that as the Reynolds number increases the impact of the wall is reduced. The Reynolds number at which the wall has an impact (which is to decrease the effectiveness compared to the ideal no-wall case) is observed to depend on the thermal utilization, the thermal mass of 


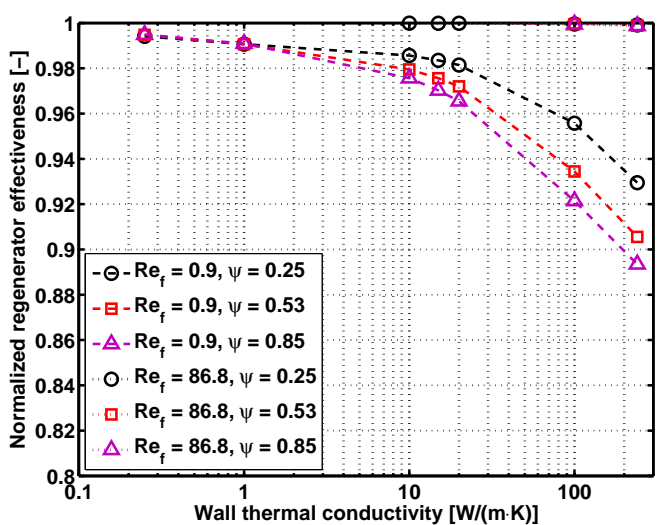

(a) $\varphi=0.5$

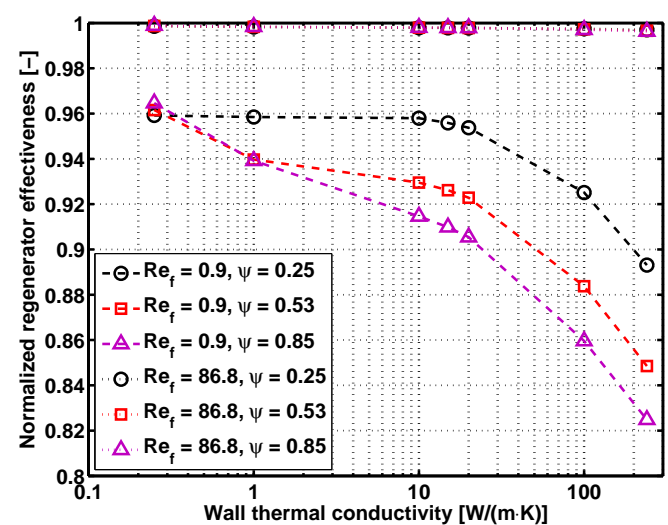

(b) $\varphi=1.5$

Figure 4: (Color online) The regenerator effectiveness (defined in Eq. 39) as a function of the wall thermal conductivity and normalized to the no wall case. The legend refers to cases with different wall thicknesses expressed as a ratio between the thermal mass of the wall and the Reynolds number $\left(\operatorname{Re}_{\mathrm{f}}\right)$.

the wall and the diffusivity of the wall. Clearly, the wall can have significant impact on performance at lower Reynolds numbers.

\subsection{Effectiveness as a function of wall conductivity}

Figure 4 presents the normalized regenerator effectiveness as a function of the thermal conductivity of the wall at different values of $\psi, \operatorname{Re}_{\mathrm{f}}$ and $\varphi$. At large Reynolds numbers the impact of the wall is minimal (as seen in Sec. 3.2). However, at lower Reynolds numbers it is seen that the impact of the thermal conductivity of the wall is significant in particular when it is greater than about one.

At the lower value of the utilization (Fig. 4(a)) the impact of the wall is generally smaller than at the higher value of the utilization (Fig. 4(b)). In the latter case the duration of the fluid blow periods is greater than in the former case and thus the time for heat transfer between the solid/fluid and the wall is greater explaining this effect. Also, in the case with a low utilization the ratio of thermal mass of the wall and the solid has an impact at values of the wall conductivity of $10 \mathrm{~W} /(\mathrm{m} \cdot \mathrm{K})$ and greater. At the larger value of the utilization this ratio $(\psi)$ is important in all cases except at the lowest value of the wall conductivity $(0.25 \mathrm{~W} /(\mathrm{m} \cdot \mathrm{K}))$. 


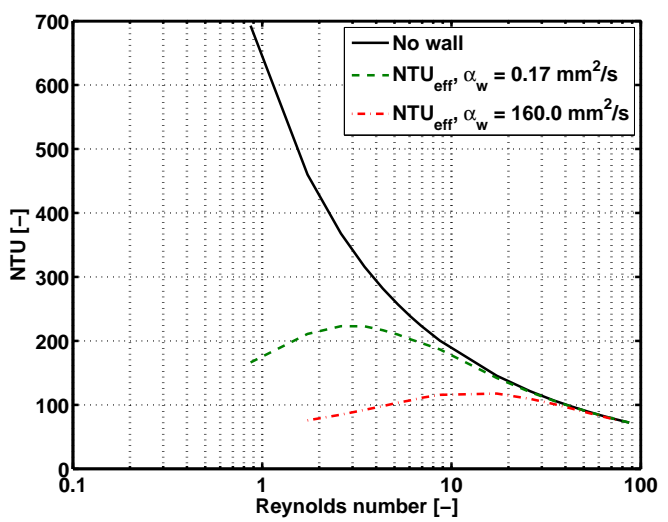

(a) $\varphi=0.5$

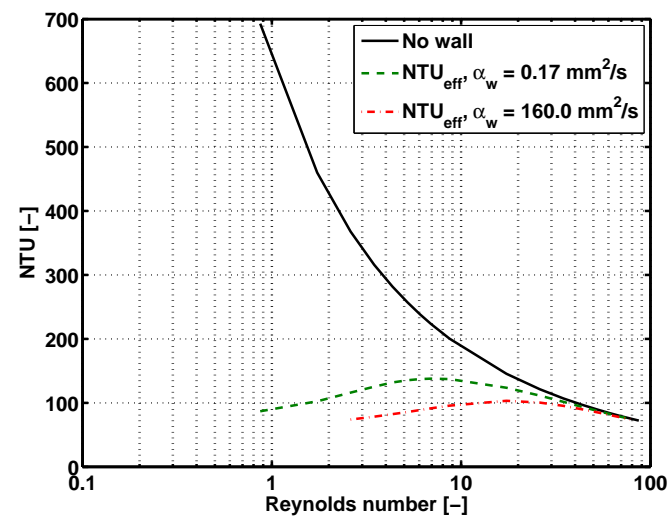

(b) $\varphi=1.5$

Figure 5: (Color online) The NTU (Eq. 37) as a function of Reynolds number. The black solid line represents the case where the wall is ignored. The other two curves represent cases where the wall is enabled having different diffusivities.

\subsection{Effective $N T U$}

The number of transfer units (NTU) is closely related to the regenerator effectiveness. An effective NTU is defined here in such a way as to allow the effect of the wall to be included approximately in simulations that do not explicitly consider the wall. Given the effectiveness of the model predicted when the wall is ignored (denoted $\epsilon_{\mathrm{nw}}$ ) it is possible to match the model results that include the wall by varying the NTU, the result referred to as an effective NTU. Denoting the effectiveness of the model when including the wall $\epsilon_{\mathrm{w}}$ this may be expressed as:

$$
\mathrm{NTU}_{\mathrm{eff}}=\mathrm{NTU}\left(\epsilon_{\mathrm{w}}=\epsilon_{\mathrm{nw}}\right) .
$$

It is assumed that the values of the effectiveness are evaluated at the same conditions (Reynolds number, bed geometry etc.). Figure 5 shows the NTU as a function of Reynolds number for the no-wall case and when including the wall (at two different values of the wall diffusivity). Again, at larger Reynolds numbers the wall has very little impact. However, as the Reynolds number is decreased the effective NTU is significantly smaller than the no-wall NTU.

\section{Discussion and Conclusion}

A numerical model that simulates the transient heat transfer problem present in a regenerator was developed, presented and validated. The model 
is two-dimensional and resolves the axial (flow) direction and the radial direction thus assuming azimuthal symmetry. Three domains are considered: the solid regenerator matrix, the heat transfer fluid and the surrounding housing or wall. Applying the model the impact of the wall on the heat transfer performance of a regenerator was quantified.

Single-blow experiments were simulated with the model by assuming a common $\mathrm{Nu}$-Re correlation and enabling the wall under various conditions. The model was then applied with the wall disabled and the Nusselt number varied in order to match the temperature outlet curve of the fluid. This resulted in a "measured" Nu-Re relation that is unphysical in the limit when the Reynolds number goes to zero because the Nusselt number becomes negative. Related problems occur when experimentally determining Nusselt numbers, as shown in Ref. [1].

The model was then applied for periodic or cyclic steady-state simulations where the regenerator effectiveness was used as a parameter for quantifying the impact of the wall under realistic regenerator operating conditions. The resulting effectiveness when taking the wall into account was normalized with the effectiveness of the ideal no-wall cases and it was shown that for Reynolds numbers below 100 the regenerator wall (housing) may have a significant impact on the regenerator effectiveness. For the present study the reduction in regenerator effectiveness compared to the case with no wall was as much as $18 \%$. It was also shown that the effect depends not only on the Reynolds number but also on the diffusivity and thermal mass of the wall as well as the thermal utilization.

Due to the close relation between regenerator effectiveness and the NTU, an effective NTU was defined for the model cases that include the wall. The effective NTU is defined as the NTU required by the case with no wall in order to match the effectiveness associated with the case with the wall included. The effective NTU is always less than the actual NTU; at low Reynolds number the reduction in the effective NTU is large indicating that the wall has a significant impact on performance.

The key conclusion from this work is that the regenerator housing should be carefully considered both when designing and interpreting experimental single-blow results with the purpose of determining heat transfer coefficients as well as when operating regenerators under periodic steady-state conditions. At low Reynolds numbers and high Prandtl numbers even thin walls with low diffusivities may influence the performance significantly.

The model was developed in Fortran and is freely available through 
Google Code [7].

\section{Acknowledgements}

K.K. Nielsen wishes to thank The Danish Council for Independent Research - Technology and Production Sciences (Contract no. 10-092791) for financial support.

\section{References}

[1] M. Frischmann, S. Jacobs, G. Nellis, and S. Klein. Measuring nusselt number using a single-blow regenerator facility. In P. W. Egolf, editor, Third International Conference on Magnetic Refrigeration at Room, pages 443-448. International Institute of Refrigeration, 2009.

[2] W. Roetzel, S. K. Das and X. Lou. Measurement of the heat transfer coefficient in plate heat exchangers using a temperature oscillation technique International Journal of Heat and Mass Transfer, 37: 325-331, 1994

[3] P.J. Heggs and D. Burns. Single-blow experimental prediction of heat transfer coefficients - a comparison of four commonly used techniques. Experimental Thermal and Fluid Science, 1:243-251, 1988.

[4] P. V. Danckwerts. Continuous flow systems. Chemical Engineering Science, 2(1):1-13,1953

[5] T.E.W. Schumann. Heat transfer: A liquid flowing through a porous prism. Journal of The Franklin Institute, 208(3):405-416, 1929.

[6] G. D. Dragutinovic and B. S. Baclic. Operation of Counterflow Regenerators, volume 4 of International Series on Developments in Heat Transfer. Computational Mechanics Publications, 1 edition, 1998.

[7] K.K. Nielsen. A 2D radial passive regenerator model including wall domains. http://code.google.com/p/passiv-regenerator-2d-radial/, November 2012.

[8] N. Wakao and S. Kaguei. Heat and Mass Transfer in Packed Beds. Gordon and Breach Science Publishers, 1982. 
[9] F. P. Incropera and D. P. Dewitt. Introduction to heat transfer. John Wiley and Sons, Inc., 3 edition, 1996.

[10] S. Ergun. Fluid flow through packed columns. Chemical Engineering Progress, 48(2):89-94, 1952.

[11] M. Kaviany. Principles of Heat Transfer in Porous Media. Springer, New York, 2nd edition, 1995.

[12] D.B. Ingham and I. Pop, editors. Transport Phenomena in Porous Media. Pergamon Press Inc, Elsevier Sciende Ltd. The Boulevard, Langford Lane, Kidlington, Oxford OX5 1GB, UK, 1 edition, 1998.

[13] G. R. Hadley. Thermal-conductivity of packed metal powders. Int. J. Heat Mass Transfer, 29:909-920, 1986.

[14] J. M. P. Q. Delgado. A critical review of dispersion in packed beds. Heat and Mass Transfer, 42(4):279-310, 2006. 